\title{
OPTICAL SPECTROSCOPY OF SIX CARBON-RICH PROTO-PLANETARY NEBULAE
}

\author{
B.J. HRIVNAK \\ Dept. of Physics \& Astronomy, Valparaiso University, Valparaiso, IN 46383, USA
}

We are engaged in a program of optical spectroscopy of proto-planetary nebulae (PPN). The objects were initially selected from the IRAS database on the basis of their strong infrared excesses, indicating dust temperature of $150-250 \mathrm{~K}$. Spectra have been obtrained at medium resolution for the purpose of obtaining the spectral type, luminosity class, and to search for chemical peculiarities resulting from the post-AGB nature of the objects. We found our PPN candidates to typically display F-G supergiant spectra.

In this paper, we discuss in particular 6 PPN which show molecular carbon, IRAS $04296+3429,05113+1347,20000+3239,22223+4327,22272+5435$ and 23304 +6147 . From our spectra, we find the following results:

1. all 6 show $\mathrm{C}_{2} \lambda 4735,4717$ (and $\lambda 5165$ when spectra cover this wavelength);

2. 4 of 6 also show $\mathrm{C}_{3}$, which had previously been reported only in AFGL 2688 and late $\mathrm{N}$-type carbon stars;

3. all 6 show strong BaII $\lambda 4554$ and strong SrII and YII (s-process elements).

Those carbon and s-process enhancements are in accord with the general expectations of the effect of thermal pulses and mass loss on the AGB, and support the post-AGB nature of the objects.

These 6 carbon-rich PPN have several other properties in common, some of which are quite rare.

1. 5 are among the small group of 9 known IRAS sources which display an unidentified emission feature at $21 \mu \mathrm{m}$

2. 3 of them have been observed spectroscopically in the $3 \mu \mathrm{m}$ region, and all 3 show the $3.3 \mu \mathrm{m}$ emission feature.

3 . 2 of these 3 are among the few sources with an unusually strong $3.4 \mu \mathrm{m}$ feature, comparable in strength to the $3.3 \mu \mathrm{m}$ feature.

4. Molecular line observations detect $\mathrm{CO}$ and $\mathrm{HCN}$ in these objects.

These and other common properties are summarized in the table. We suggest that the carbon richness and low stellear temperatures $(\mathrm{T}=4500-6000 \mathrm{~K})$ are important parameters in relating these properties. 